\title{
Algorithmic Ecologies: Integrating Dynamics and Real- time Simulation Feedback for Design Optimization
}

\author{
KELLY WINN \\ Rensselaer Polytechnic Institute
}

To meet and respond to future challenges posed by large scale climate crises before they occur, it is necessary to begin addressing the adverse effects of the built environment by developing biological buffers or designing new systems to restore, replenish, and remediate ecological systems. Designers must pursue innovative methods to model, analyze, simulate, and optimize these systems, in order to design architecture and landscape systems that respond effectively to complex ecological problems occurring over significant temporal and geographic scales. As a result, data driven design is required to balance competing design criteria effectively through trade-offs to meet design objectives of high-performance architectural systems and is increasingly a requirement of design practice for the foreseeable future. This type of advanced computational design requires sophisticated models to be developed integrating these complicated tools in the design process for simulation and dynamic modeling. This type of advanced computation requires making use of readily available datasets defining weather profiles, material properties, energy performance, building physics, etc. that can all be used to inform the design process and test the suitability of design solutions. In this paper, a process for integrating multi-objective genetic algorithms (MOGA) for design optimization driving parametric models and environmental simulation is discussed. Three student case studies from a two-semester course sequence focusing on ecology-centric design schemes are presented, demonstrating the design optimization of sufficiently complex parametric models. These case studies will be used to demonstrate the potential for such methods to be incorporated in different aspects of the conceptual design process and architectural course curriculum.

\section{INTRODUCTION}

Advanced computational methodologies are becoming a ubiquitous presence in the practice of architecture. With the recent occurrence of large scale climate crisis, from storm surge to coastal flooding to water scarcity to contaminated ecosystems, a noticeable shift is taking place away from the traditionally anthropocentric frame of architecture towards one of environmental responsivity and a directed ecologycentric focus. In order to design for increasingly more polluted environments, it is necessary to gage long-term effects, and seek solutions to remediate, filter, or reconstruct damaged and lost habitat. Living systems and natural processes typically occur on much longer time scales and are more sensitive to long-term shifts in climate or environmental context than are normally considered within architecture or landscape design. Long term systems outside of normal human spatial or temporal scales, such as representing life cycles or global energy flows, can be difficult to assess with existing models or methods for design. [1] This suggests anticipating forces that cannot be readily observed by human senses, requiring the use of sensors to gather empirical data over time in the physical world, or developing predictions based on sophisticated computer models and simulation in a digital environment. This concept of an ecosophical approach to design [2]; as spatial environmental experiences, reclaimed ecological territories, and performative environmental systems (Figure 1); has led to a similar shift in practice towards environmental performance, ecology and autonomous design, driving the introduction of new methods to analyze and develop solutions to the types of complex problems posed by ecologycentric design. In preparation for the emerging challenges of a changing design profession, it is necessary to be able to understand and integrate multiple streams of climatological and environmental analysis data in the design process, so that better assumptions can be made about design performance and the long-term effects of the built environment on ecological systems. As a result, simulation and data analysis techniques are becoming increasingly more important to the realization of holistic design projects. This increasing role of performance criteria in the design process, such as energy efficiency and environmental performance, requires better computational tools for building energy modeling and building information modeling to validate design assumptions. This research investigates the application of an algorithmic design-optimization process by applying genetic algorithms in the analysis of complex modeling operations, and integrating advanced simulation techniques during preliminary schematic design phases, where the greatest performance benefits can be achieved [3].

\section{BACKGROUND}

The work discussed here explores several biological and ecological analogues explored by students as case studies of form expressed as computational morphology for the application of multi-objective genetic algorithm (MOGA) design-optimization, representing a continuation of work seen previously in [4] and [5]. Like these previous examples, these geometric studies are pursued as highly rationalized systems for complex interactions of energy and material exchange present in natural systems. Through an intelligent aggregation of material, the performative characteristics of 

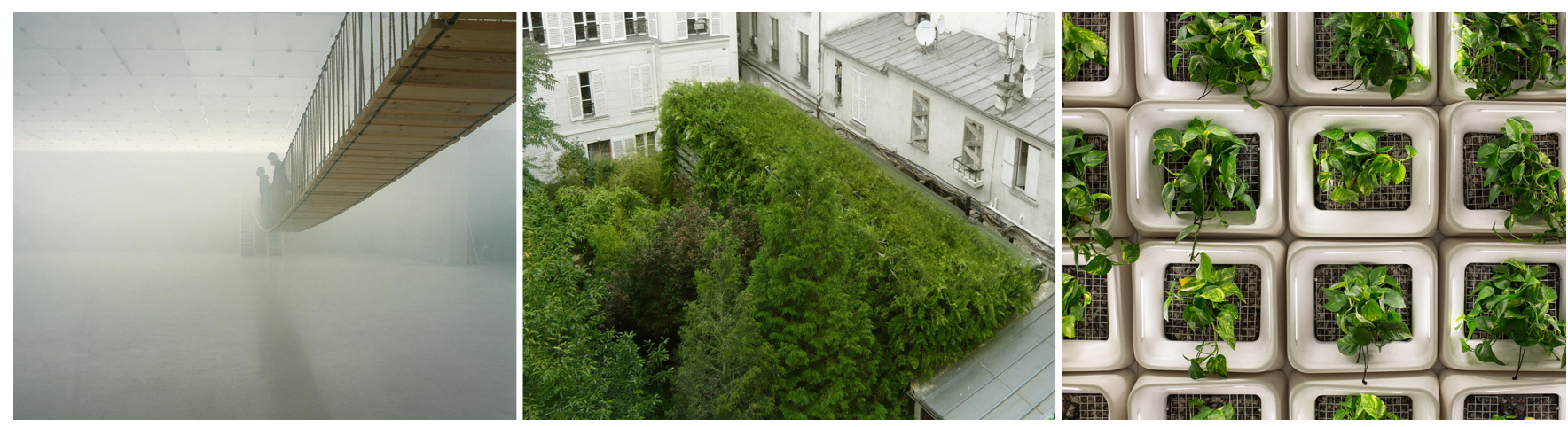

Figure 1: The Mediated Motion weather system [6], Lost in Paris green façade/landscape [7], green wall for indoor air phytoremediation [8].

surfaces and material systems are creatively controlled to promote different structural logics, thermodynamic properties, or fluid dynamic affects. Since these geometries are produced by algorithmic operations, the parametric inputs controlling these models are flexible and can be efficiently modified autonomously by an optimization process. This does not mean that the designers must entirely relinquish control to an algorithmic process. In fact, design optimization is a highly deterministic process that can have very different outcomes based on the subjective values of the individual programmer when selecting criteria to be given priority in the model. Simply favoring one set of criteria over another can result in dramatically different solutions, both quantitatively and qualitatively.

Rather than pursuing a more subjective notion of performance, typical to most architectural studio courses, a more straight-forward rule based approach to design [9] is pursued as a fluid segue into algorithmic design analysis and optimization during an introductory course on parametric modeling. By establishing straight-forward and repeatable rules for the design process, the task of ascribing genetic algorithms to the process during the second semester course is simplified for students. From an ecological perspective, it is necessary to step outside of the typical anthropocentric focus by approaching the design process without preconceptions, defaulting to typical practices, or relying on design tendencies, by instead allowing the simulation to derive as many solutions as possible that may likely have gone unconsidered; rather than trying to approach the process with a preconceived notion of a "preferred outcome" and attempt to "force" a simulation to confirm a desired solution that has already been predetermined. It is necessary to resist the tendency to pursue initial aesthetic solutions that are perceived as optimal geometries, and instead actively test potential geometric solutions through computation to understand and predict future performance. In order to move from an area of design where solutions are assumed to work into a domain of reasonable expectations that a given design will perform as desired, design optimization processes can be useful tools to align expectations and supply much needed feedback to the design process. Unlike a completely subjective design process that only prioritizes the concerns of the designer, a synthetic design process provides the designer with necessary resistance to balance personal preferences against design requirements.

\section{METHODS}

For these studies, a framework was established for simulating architectural design conditions, and assessing useful design performance metrics. With the intention to extract a useful design analysis from the simulation, an appropriate test for "fitness" must first be established. If the objectives of the optimization study are two simple or needlessly complex, appropriate solutions cannot be reached successfully. For this reason, it is important to carefully select and define fitness values and limit the bounds of the simulation. Once the model is setup correctly, an optimization process can be run to generate a solution. (Figure 2)

Before connecting an evolutionary solver to the model, a graphical matrix is established representing the range of model variability. This allows the bounds of the study to be clearly defined, as well as run a first-pass test of the full range of geometry in the model looking for major errors or excluding invalid geometry that will disrupt the function of the evolutionary solver during the simulation. Once troubleshooting is complete and the model is successfully tested, the input variables and output fitness values can be attached to the evolutionary solver. Once the simulation begins the solver runs the genetic algorithm and tests possible solutions in terms of fitness. Here, the Octopus evolutionary solver component for the Grasshopper plugin is used instead of the native Galapagos solver, so that multiple fitness values can be brought in to run a MOGA process and multiple variables can be tested against each other during the optimization process. [10] The first design optimization process is then allowed to run for several generations at a reasonable population size, until a Pareto Front can be observed in the graphical interface.

It is important to start with enough parametric flexibility in the original model so that issues can be clearly identified, and parameters of the simulation can be narrowed and refined in 


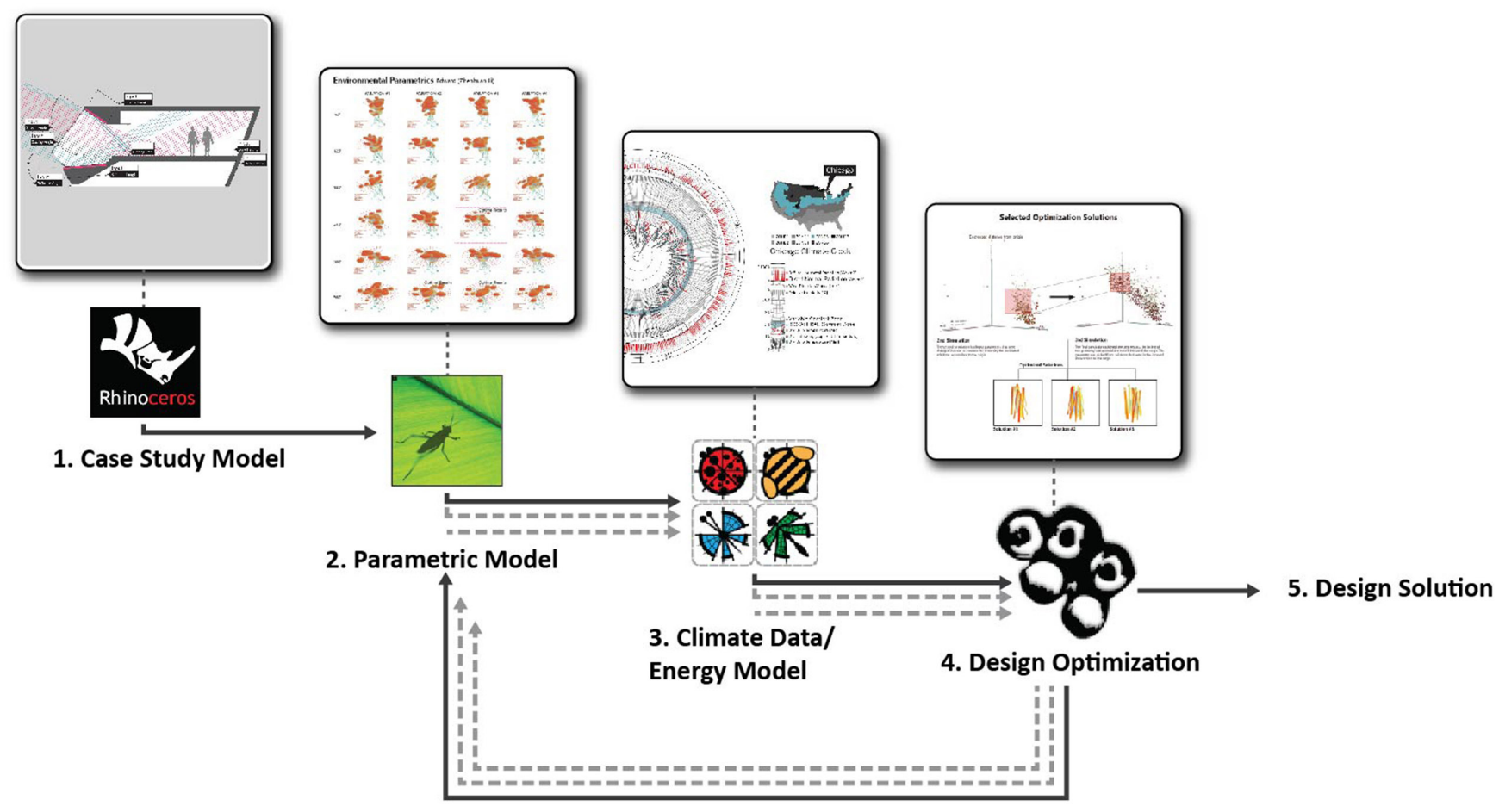

Figure 2: Showing an iterative process for form generation, data analysis and design optimization.
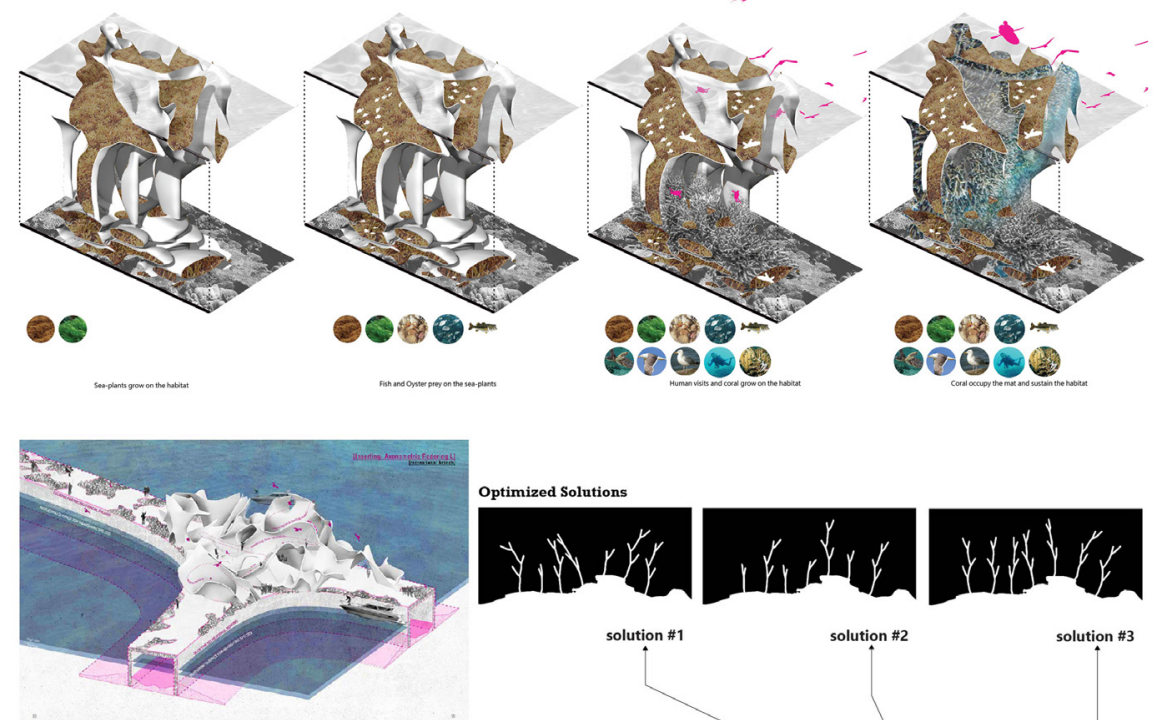

Optimized Solutions
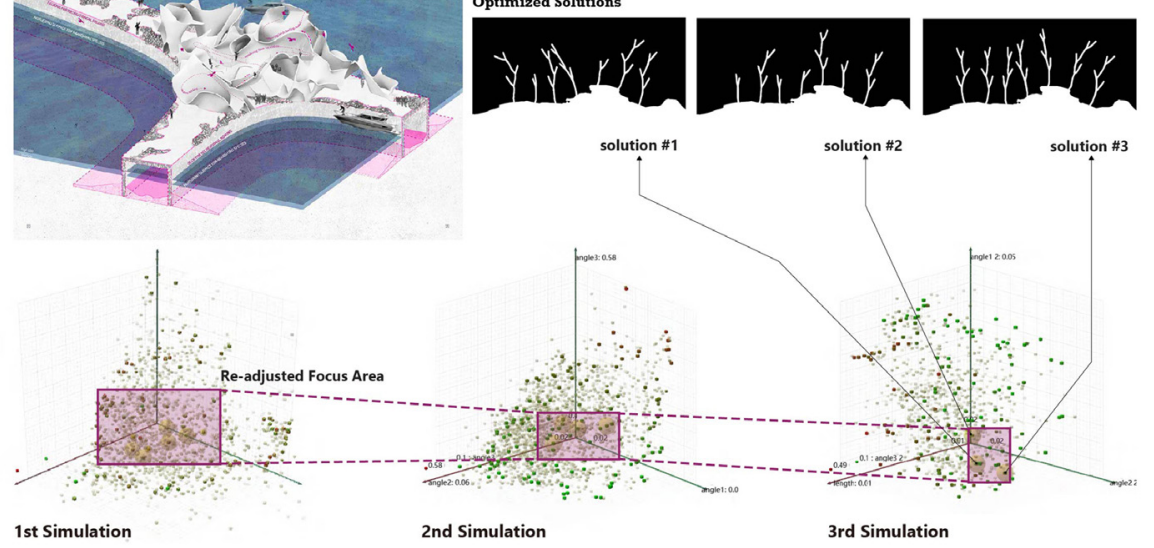

Figure 3: Bio-inhabitation of the artificial reef structure over time by oceanic flora and fauna (top). Design optimization process of the overall reef structure in relation to local ocean currents and distribution of I-system geometry (bottom). Student: Xiaoyu Wu. 

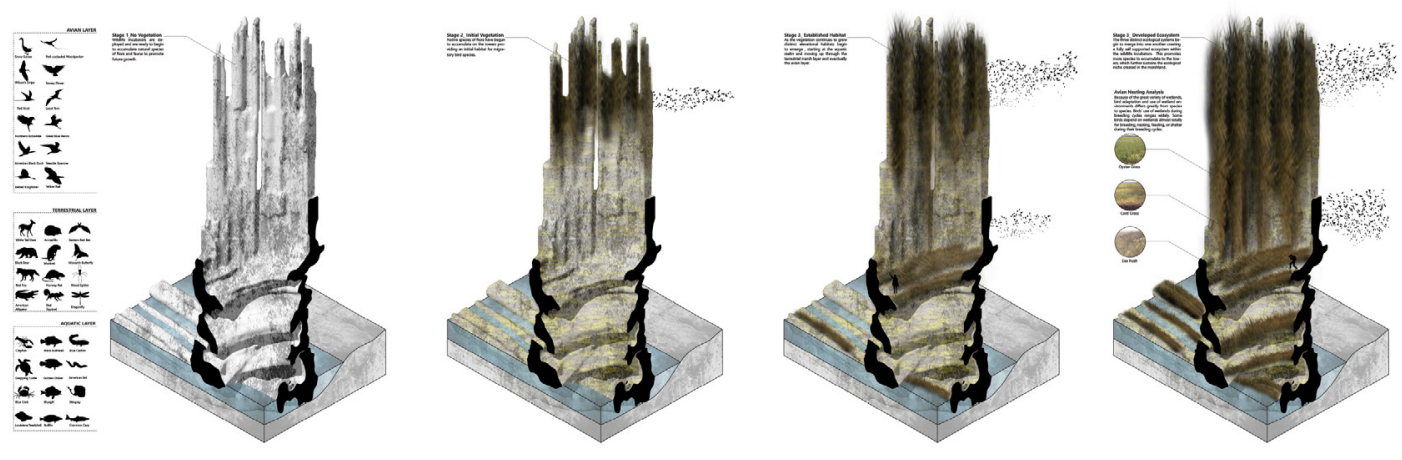

Figure 4: Visualization of ecological inhabitation over time (top), 3D printed design concept model ( bottom left). Design optimization process for interlocking columnar geometry (bottom right). Student: Milton Major.
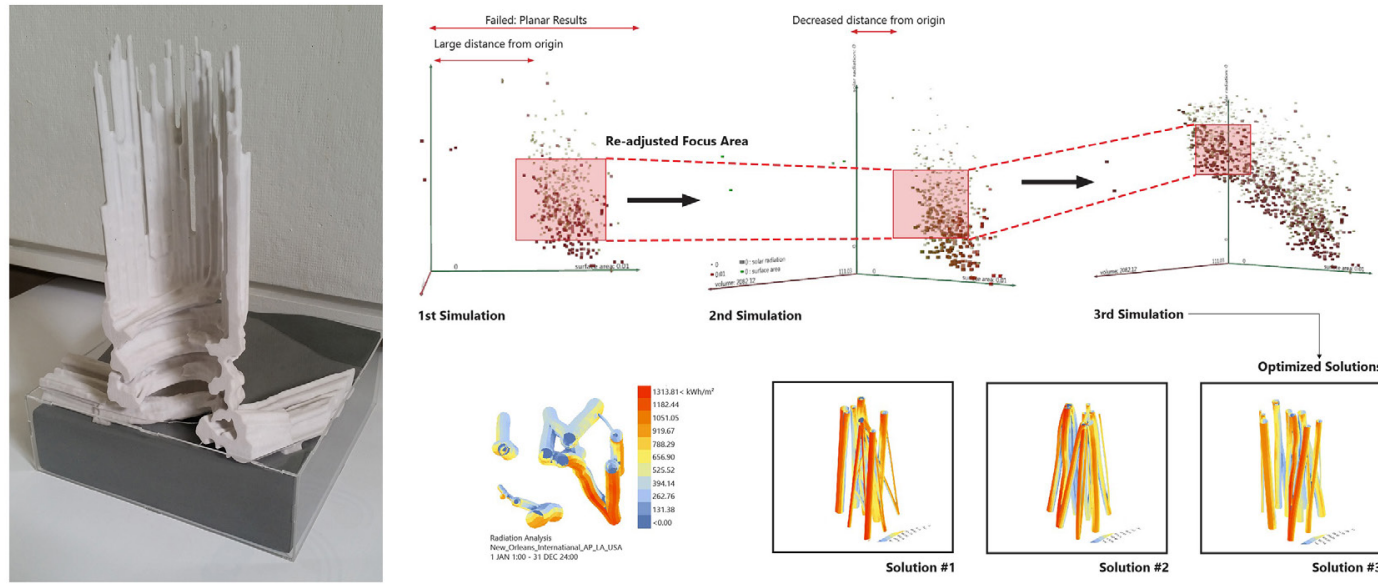

3rd Simulation
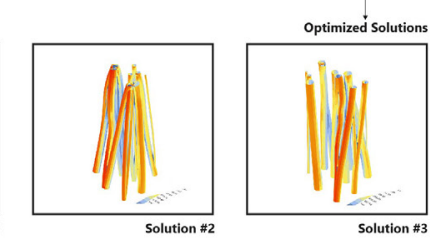

Student: Milton Major.

future iterations. During this first pass, negative results are not only useful but also desirable, revealing problems with the simulation and helping to troubleshoot issues that might become greater problems later-on. Since the simulation is running a minimization process through the Octopus solver, solutions closest to the origin on the graph are then selected and the parameters of these solutions are used to reduce the global geometric range of the parametric model, so that the next optimization process can be run with a tighter simulation focus. By first starting with a loose geometric range, the evolutionary solver is allowed to direct the simulation focus towards the best possible range solutions, rather than being constrained around a desired outcome from the start by the person running the simulation. This also helps avoid common problems that can be avoided with a good simulation setup, providing a reasonable degree of confidence that the solution will fall within the parameters of the simulation and can be correctly determined [11]. This preliminary study is focused on developing a clear and iterative parametric framework, which can be built upon in the future, representing the three fundamental steps in the design process: Design --> Analysis --> Synthesis. The optimization process is then run at least two to three more times, adjusting the parametric range and extents of the simulation with each pass, ultimately resulting with a good selection of solutions within the optimal range of performance sought by the study.

\section{FILTERING REEF STRUCTURE FOR COASTLINE RESTORATION}

In this case study, a deployable system for coral reef restoration is proposed to restore portions of reef or other costal ecosystems that have been devastated by coral bleaching or other increasingly prevalent climate crisis events. Artificial reef systems studying the biomimetic growth of reaction diffusion geometries studied previously by [12] and differential growth geometries of coral [13], were investigated as an efficient double-sided surface geometry for restoring reef systems, by providing surface area for sea life to anchor themselves to while creating an ecological buffer, filtering and cleaning contaminants from the ocean. These complex geometries were then developed to provide a maximum amount of available surface area for inhabitation by various species of corals and oysters to reinforce the structure and provide habitat for other forms of aquatic sea life. In order to distribute these reef structures effectively and interact with a maximum area along a coastal system, A series of L-system algorithms were selected and programmed into the model for the macro geometry of the reef system as semi-random branching reef structures arrayed along a site on the Alaskan coastline. These geometries were then used as the focus of the optimization process to increase reef area while avoiding collisions in the distribution of these branches, and maximize the interaction of the system with ocean currents for filtration as an ecological buffer. The MOGA optimization process 

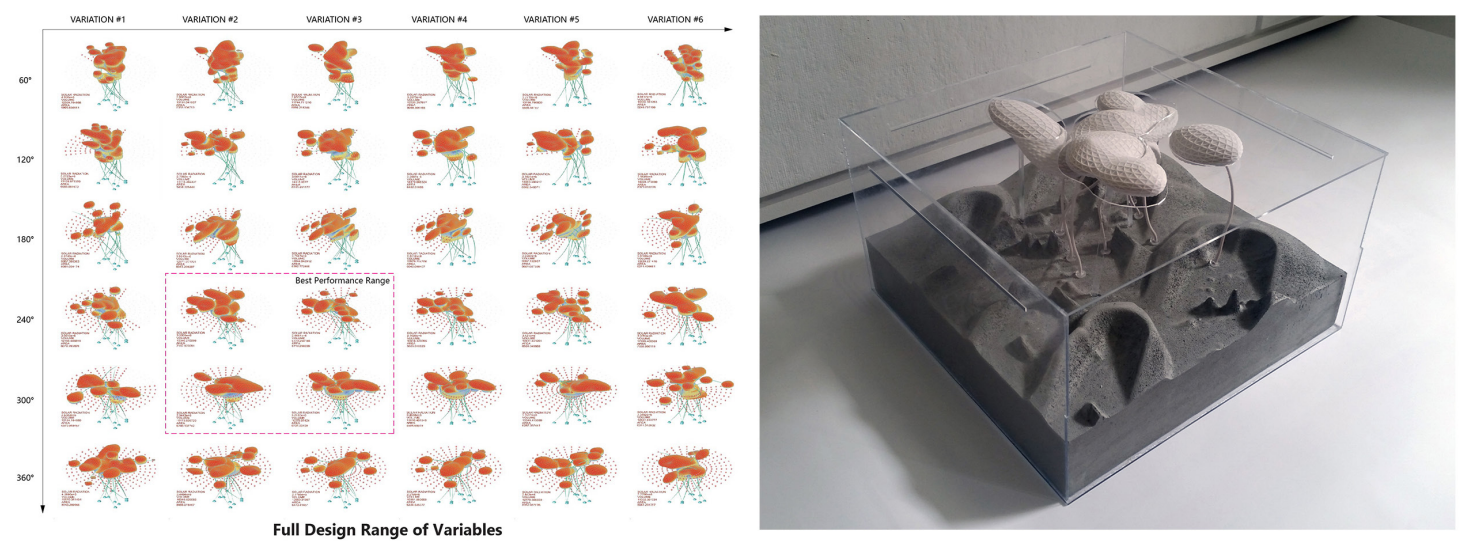

Figure 5: Showing the original range of geometric variability of the parametric model (top left). 3D printed model of final selected geometry (top right). Multi-stage design optimization of deployable floating structure showing selected final optimal solutions (bottom). Student: Zhenhuan Li.

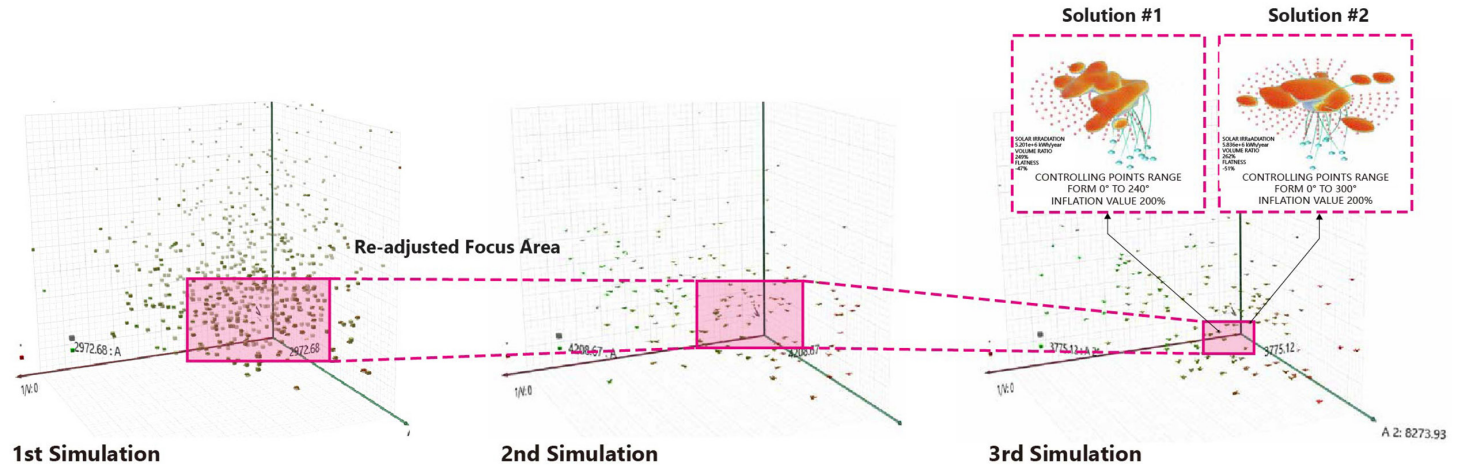

was then applied to the analysis of the overall form of these branching reef structures. In this case, each reef structure was given a unique I-system definition and the optimization process was used to determine the individual parameters of each I-system to avoid conflicts, rather than using one singular definition that would simply branch without collisions (Figure 3). Input parameters for adjusting geometry included: branching angles, branch growth length, and vectors of growth. These input parameters were then attached to the multi-objective evolutionary solver, and performance values were selected to assess the fitness of solutions generated by the parametric model; such as angles of incidence between branches and coastal wave patterns, structural proximity, and total surface area.

\section{NESTING HABITAT FOR MIGRATORY BIRDS}

Due to increasing urban development, especially along coastlines, habitat loss for migratory birds is an increasing concern. In this case, providing vertical nesting area separate from human intervention that could develop and add biological diversity over time was selected as an ideal morphological solution to this problem. For this study agent-based vector paths were applied as a generative process for form development, similar to the method in [14], resulting in a tower structure separating human visitors to the site from migratory birds nesting at the top of the structure. In this case study, agent vectors are attracted to central gravitational point before being drawn along a vertical vector creating a vertical structure reminiscent of volcanic structures observed in basalt columns.

Due to the verticality of the final form, structural integrity was of particular concern, this criterion was then balanced against aesthetic desires for openness in the system, as well as allowing solar access down into the center of the form. To optimize the structural networks between columns and balance the structural concerns against competing aesthetic and ecological criteria, the genetic algorithm was applied to find optimal structural solutions. To resolve the structural logics of the form, a novel application of dynamic simulation and real-time physics simulation was integrated into the design optimization process. In this case, a dynamic physics model was developed using the Kangaroo Physics component to allow columns to be drawn together dynamically in places of proximity due to attracting forces during each iteration. Additionally, simulating solar exposure of the resulting geometry were incorporated into the workflow similar to [15]. The graphs comparing structural interconnectivity, openness, and solar exposure from the iterative optimization process, along with the geometries resulting from the study can be seen in Figure 4. 


\section{DEPLOYABLE BIOREMEDIATION ISLAND}

Another ecological concern with polluted ecosystems is the challenge of filtering contaminants in post-industrial areas, e.g. industrial pollutants such as Polychlorinated biphenyls (PCBs) that build up in riverbeds, which represents a significant risk to certain species of fish making them unsafe for human consumption. For a design concept focused on removing PCBs from contaminated riverbeds, a structural logic to generate form for floating islands was developed based on a radial cluster of curves as a framework for generating the globular geometries seen in Figure 5. This potential solution based on the concept of a floating archipelago of bioremediation islands, similar to systems used to dredge artificial islands, would vacuum sediment from the riverbed and employ plants and biological agents to break down harmful chemical compounds and remediate the contaminated soil for reintroduction to the riverbed or for agricultural use. The curves used to derive geometry were constructed along a series of anchor points used to generate semi-random geometries. An analysis matrix representing the range of geometry possible in the parametric model can also be seen at the top left in Figure 5. As the anchor points are adjusted at random, new formal possibilities are generated and then analyzed for flatness, solar exposure, and volume in the three stages of the iterative design optimization process shown at the bottom of the Figure. The optimization process allows for hundreds of tests of intermediate geometries using a genetic algorithm to narrow down the range of possibilities to a small focus area.

\section{RESULTS AND DISCUSSION}

The work discussed here represents results from a twosemester course sequence focused on ecological design and parametric computation. This course sequence poses a fundamental question, "how can you determine quantitatively if a design is successful?" This is quite different than asking if a design is pleasing. Simply by asking if a design will in fact produce the results it promises and perform as expected, has a dramatic effect on the design process. It is common practice to speculate on the implications of a given design; however, it is a significantly more difficult challenge to show that a design will operate as proposed. This by necessity requires a combination of environmental simulation and iterative design, to allow simulated performance values to invalidate design solutions. Instead, students must actively work to correct problems and continuously refine geometry until a successful solution is reached. Using project work from design studios as case studies, students are introduced to advanced computational design techniques and methods for analysis and simulating design performance.

It can be difficult for students to quickly pick-up the necessary skills to apply basic environmental simulation to their own work, while adapting to a more rigorous design process required to produce repeatable results and develop working design optimization models. Students must be prepared to set rules for their own design process, and adhere to those rules closely enough to reproduce results. This can be difficult for students familiar with more subjective design processes. Similarly, a base understanding of specific knowledge related to the ecological design focus, as well as knowledge specific to the environmental simulation or any metrics a given simulation is calculating must first be understood in order to avoid common errors and interpret results. To overcome such a steep learning curve, a two-week boot camp is necessary to begin the course sequence to introduce students to the software and the possibilities and the capabilities the software offers, so that many common obstacles to developing a working fluency with the software can be avoided. This allows students to steadily build a fundamental level of comfort with the process on a day to day basis rather than forgetting important content during the week to week delay required for a semester long course.

Once students have an idea of what the software is capable of they can return to a technique or method and figure out a specific script as the need arises. Since it is easy to listen to an explanation of a given script and quickly forget that knowledge after it is no longer useful, it can be difficult for students to develop in-depth comprehension until they have made use of that script in their own design work. For this reason, it is more important to give students a varied and detailed overview, so that they can start beginning to envision uses and applications for the software platform, that can then be explored in detail as necessary. Working with a computer simulation and allowing an algorithm to calculate a solution and guide the design process can be frustrating. However, once such a connection is made between the process and an actual design problem, and the software is used to greatly improve the efficiency of completing a challenging task or reduce time commitments for difficult modeling operations, then the software becomes invaluable, and designers will explore the software farther than they would have initially during a more formal tutorial. This proved to be a significantly more successful format than a drawn out weekly course. If students are not building competency in the software daily, especially at the introductory level, it is quite easy to forget any new skills they learned during a single class period waiting an entire week before returning to the material during the next class in a weekly course format. Finally, the course sequence is followed up with a seminar on design-optimization, once they have developed a foundation with the subject matter.

\section{CONCLUSION}

In order to design outside of the typical anthropocentric focus of architecture, it is necessary for designers to incorporate methods that shift the focus of design towards longer term design performance and challenge the personal and aesthetic preferences of the designer. In this way, design optimization can be antagonistic to typical design traditions, requiring an atypical iterative design process to effectively control the simulation and respond to specific performance criteria. As seen with 
the three case studies discussed, MOGA optimization can be applied in significantly novel ways to effectively provide important data feedback for many aspects of a given design concept at any stage of development in a design studio course sequence, especially during schematic design phase where designers have more flexibility with massing and overall geometry. As a tool for informed design, this allows designers to assess complex environmental performance and ecological problems that cannot be effectively addressed with more traditional design procedures. It can be difficult for designers to relinquish this much control over the design process. Design optimization depends on variability, so if an optimization is restrained and there is no wiggle room for the computer to find a different solution, and any results may ultimately only reinforce the assumptions of the programmer; however, benefits of pursuing such a process can be seen in the final design and easily understood. Essentially, by allowing the computer to invalidate design assumptions, new and innovative design solutions can emerge unexpected, which meet initial design requirements while providing higher levels of performance. Rather than trying to force poor performing design concepts developed primarily for pragmatic and aesthetic solutions to perform efficiently, simulation can be used to provide designers with real time feedback on the impact of their design decisions on multiple metrics of performance. This shows the opportunity for data driven design to help avoid many of the common problems inherent in traditionally anthropocentric design processes, and the potential for finding solutions to emerging challenges facing the built environment.

\section{ENDNOTES}

1 Morton, T. Hyperobjects: Philosophy and Ecology After the End of the World (Minneapolis: University of Minnesota Press, 2013).

2 Roche, F. "(Science) Fiction, Ecosophical Apparatus and Skizoid Machines." Architectural Design, Volume 82, Issue 2 (John Wiley \& Sons Ltd., 2010), 64-71.

3 Lechner, Norbert. Heating, Cooling, Lighting: Sustainable Design Methods for Architects, 4th Edition (Hoboken: Wiley, 2014).

4 Cupkova, D. and Azel, N. "MASS REGIMES: Geometrically Actuated Thermal Flows." Paper presented at the ACADIA 2014 Conference (Los Angeles: October 23-25, 2014).

5 Winn, K. Inter-scalar Multivariable Decision Making Framework for the Architectural Envelope (Troy: Rensselaer Polytechnic Institute, 2014).

6 Studio Olafur Eliason. The Mediated Motion (Kunsthaus Bregenz, Austria: 2001).

7 R\&Sie(n). Lost in Paris (Paris, France: 2008).

8 Center for Architecture Science and Ecology (CASE). “Green wall." Public Safety Answering Center II (Bronx, NY, 2016).

9 Wolfram, Stephen. A new Kind of Science. Champaign: Wolfram Media, Inc. Accessed May 19, 2017. https://www.wolframscience.com/nksonline/toc.html

10 Robert Vierlinger "Octopus" University of Applied Arts Vienna. Accessed May 19, 2017. http://www.food4rhino.com/app/octopus

11 Rutten, David. "Evolutionary Principles Applied to Problem Solving." Paper presented at the Advances in Architectural Geometry 2010 Conference (Vienna: September 18-21, 2010).

12 Erioli, A. and Zomparelli, A. "Emergent Reefs" Paper presented at the ACADIA 2012 Conference (San Francisco: October 18-21, 2012).

13 Nervous System "Floraform" Accessed May 19, 2017. http://n-e-r-v-o-u-s.com/ projects/albums/floraform-inspiration/

14 Tsiliakos, M. 2012. "A multi-agent approach to stress driven material organization" Paper presented at the eCAADe Conference (Prague: September 12-14, 2012).

15 Roudsari, Mostapha. "Ladybug: A Plugin for Environmental Analysis." Presentation to the Building Sciences Lecture Series (March 7, 2017). 